\title{
Type II GaSb/GaAs Quantum Rings with Extended Photoresponse for Efficient Solar Cells
}

\author{
P. J. Carrington ${ }^{* a}$, D. Montesdeoca ${ }^{\text {b }}$, H. Fujita ${ }^{d}$ J. James $^{\mathrm{b}}$, M. C. Wagener ${ }^{\mathrm{c}}$, J. R. Botha ${ }^{\mathrm{c}}$, A.R.J. Marshall ${ }^{\mathrm{b}}$, and \\ A. Krier $^{\mathrm{b}}$
}

(a) Department of Engineering, Lancaster University, Lancaster, LA1 4YW, UK

(b) Physics Department, Lancaster University, Lancaster LA1 4YB, UK

(c) Department of Physics, Nelson Mandela Metropolitan University, Port Elizabeth, South Africa

(d) Asahi Kasei Corporation, 2-1 Samejima, Fuji-city, Shizuoka 416-8501, Japan

\begin{abstract}
The introduction of GaSb quantum dots (QDs) within a GaAs single junction solar cell is attracting increasing interest as a means of absorbing long wavelength photons to extend the photoresponse and increase the short-circuit current. The band alignment in this system is type-II, such that holes are localized within the GaSb QDs but there is no electron confinement. Compared to InAs QDs this produces a red-shift of the photoresponse which could increase the short-circuit current and improve carrier extraction. GaSb nanostructures grown by molecular beam epitaxy (MBE) tend to preferentially form quantum rings (QRs) which are less strained and contain fewer defects than the GaSb QDs, which means that they are more suitable for dense stacking in the active region of a solar cell to reduce the accumulation of internal strain and enhance light absorption. Here, we report the growth and fabrication of GaAs based p-i-n solar cells containing ten layers of GaSb QRs. They show extended long wavelength photoresponse into the near-IR up to $1400 \mathrm{~nm}$ and enhanced short-circuit current compared to the GaAs control cell due to absorption of low energy photons. Although enhancement of the short-circuit current was observed, the thermionic emission of holes was found to be insufficient for ideal operation at room temperature.
\end{abstract}

Keywords: Solar Cells, GaSb, Molecular beam epitaxy, Photoluminescence

\section{Introduction}

There is currently great worldwide interest in introducing quantum dots (QDs) into solar cells (SCs) to increase the photovoltaic efficiency through the absorption of infrared radiation. One approach is the QD intermediate-band solar cell (IBSC), which is theoretically predicted to reach efficiencies up to $63 \%^{1}$. Here electrons are optically pumped from the valence to the conduction band via the QD intermediate band through the absorption of two photons which are below the band gap of the host semiconductor. Another approach is to insert QDs into the GaAs section of a triple junction solar cell to extend the absorption spectrum to longer wavelengths and reduce the band gap towards $1 \mathrm{eV}$ to achieve current matching ${ }^{2}$. To date, there have been several reports of InAs/GaAs ${ }^{3}$, InAs $/ \mathrm{GaAsN}^{4}, \mathrm{InGaAs}_{\mathrm{GaAs}}{ }^{5}$, InAs/AlGaAs ${ }^{6}$ and InAs/GaAsSb ${ }^{7}$ QDs. Previously we reported on GaAs SCs containing stacked layers of GaSb/GaAs quantum rings (QRs) $8,9,10$. These are unique compared to conventionally grown Stranski-Krastanov (SK) based InAs/GaAs QDs. Firstly, the type-II band alignment provides strong hole confinement $(600 \mathrm{meV})$ but no electron confinement, which could improve carrier extraction, prevent weakening of the built-in electric field and produce a red-shift of the photoresponse, thus increasing the short-circuit current. Secondly the use of GaSb QRs instead of QDs reduces the net strain associated with the large lattice mismatch $(7.8 \%)$, enabling multiple layers to be grown without generating threading dislocations or relaxed QDs. Finally, the incorporation of type-II GaSb QDs/QRs in GaAs has been proposed as a promising system to realize an IBSC $^{11}$. The spatial separation of electron and holes provides an IB closer to the theoretical optimum and reduces thermal emission and capture processes. The resultant GaSb/GaAs QR SCs displayed improved efficiency at longer wavelengths extending out to $1400 \mathrm{~nm}$, where the 1 sun short-circuit current density $\left(\mathrm{J}_{\mathrm{sc}}\right)$ was enhanced by nearly $6 \%$ compared to the GaAs control cell. In this work, we provide a detailed study on the temperature dependent properties of

*p.carrington@lancaster.ac.uk 
GaSb/GaAs QR SCs and elucidate important new information about the dominant recombination parameters which determines their potential and suitability for future concentrator applications.

\section{Molecular Beam Epitaxial Growth of GaSb/GaAs Quantum Rings}

A QD SC requires multiple layers of QDs in the active region to provide sufficient light absorption. However the large mismatch (7.8\%) makes stacking layers of GaSb/GaAs QDs difficult, leading to the formation of relaxed QDs containing defects and to the generation of dislocations due to the build-up of internal strain. Further difficulties include strong group$\mathrm{V}$ As-Sb exchange reactions during the capping procedure which can result in significant ring intermixing and dissolution, i.e., the volume of the rings are reduced substantially resulting in a thin GaAsSb layer and weak photoluminescence (PL) and photoresponse. The As-Sb exchange also creates a Sb floating layer at the growth surface, which can aggregate into further layers of QDs as they are stacked. This creates an inhomogeneous ring distribution where the size and density of the QDs increases up the stack, increasing the strain which generates threading dislocations. This problem was overcome through the use of a 'cold-cap' procedure to grow GaSb QRs rather than QDs ${ }^{12}$. Here the QDs/QRs are capped at a low growth temperature $\left(\right.$ at $430^{\circ} \mathrm{C}$ ) which reduces As-Sb exchange and $\mathrm{Sb}$ segregation. In the QR structures, Sb is redistributed from the centre of the dot towards the edge to stabilize the structure and reduce the net strain. Hence GaSb QRs contain fewer intrinsic defects than the GaSb QDs, making it possible to stack multiple layers in the solar cell to reduce the accumulation of internal strain. Also, despite the type- II band alignment, the reduced strain around the QR allows the electron to reside near or inside the $\mathrm{QR}$ in close proximity to the confined holes which increases the exciton oscillator strength required for strong light absorption.

\section{Solar Cell Growth and Fabrication}

SCs containing GaSb QRs were grown using a VG-V80H MBE reactor. A schematic of the structure is shown in figure 1a and an energy band diagram in figure 1b. A $3 \mu \mathrm{m}$ thick n-type GaAs:Te layer was grown first at $570^{\circ} \mathrm{C}$ with a doping density of $10^{17} \mathrm{~cm}^{-3}$. This was followed by an intrinsic region containing ten GaSb QR sheets, a $0.5 \mu \mathrm{m}$-type GaAs:Be layer with doping density of $2 \times 10^{18} \mathrm{~cm}^{-3}$, followed by a $30 \mathrm{~nm} \mathrm{Al} \mathrm{Al}_{0.8} \mathrm{Ga}_{0.2}$ As window layer and a $40 \mathrm{~nm} \mathrm{GaAs} \mathrm{cap.} \mathrm{A} \mathrm{p-i-n}$ GaAs control cell was also grown with a $400 \mathrm{~nm}$ intrinsic region which contained no QRs. The QRs were grown using the following procedure: the temperature was reduced under an As flux to $480^{\circ} \mathrm{C}$, then the GaAs surface was exposed to an antimony flux for 30 seconds creating an efficient As-Sb exchange reaction to form a thin $\sim 0.5$ monolayer (ML) of GaSb layer. Following the exchange procedure, $\mathrm{GaSb}$ is directly deposited using a growth rate of $0.3 \mathrm{ML} / \mathrm{s}$, producing nominally 2.1 ML of GaSb. The formation of the QRs was detected by a change in the RHEED pattern from streaky to spotty after the deposition of approximately 1.3 ML of GaSb. The QRs have approximate sizes of $23 \mathrm{~nm}$ outer diameter, $10 \mathrm{~nm}$ inner diameter and $1.7 \mathrm{~nm}$ height (figure 1c). Excellent structural quality is observed in each sample with no threading dislocations. The ring density per layer is approximately $1 \times 10^{10}$ rings $/ \mathrm{cm}^{2}$ with no significant variation in size or density in the separate layers. The temperature was then reduced to $430^{\circ} \mathrm{C}$ under an $\mathrm{Sb}$ flux and capped with a $5 \mathrm{~nm}$ thin $\mathrm{GaAs}$ layer. The temperature was then increased to $570^{\circ} \mathrm{C}$ under an As flux to grow the GaAs spacer layers $(35 \mathrm{~nm})$. The structures were processed into $3.5 \mathrm{~mm}$ diameter circular SCs with anti-reflection coatings (figures $1 \mathrm{~d}$ and $1 \mathrm{e}$ ). The spectral response of the SCs was measured using a $100 \mathrm{~W}$ tungsten-halogen light source through a 1/4 metre monochromator. Current density $(\mathrm{J})$ - voltage $(\mathrm{V})$ curves were performed under 1 sun illumination using a $150 \mathrm{~W}$ Oriel solar simulator.

\section{Photoluminescence from stacked layers of GaSb/GaAs rings}

Figure 2a shows the power dependent $4 \mathrm{~K}$ PL spectra from samples containing ten stacked layers of GaSb QRs. Two peaks can be clearly identified, one at an energy of $1.32 \mathrm{eV}$ from the wetting layer peak and a peak around $1.00 \mathrm{eV}$ corresponding to recombination of holes localized in the GaSb QRs with electrons in the surrounding GaAs matrix. Figure 2(b) plots the change in emission energy over six orders of magnitude of laser power. In both cases a strong blueshift of the QR emission peak is observed. Such blueshifts are characteristic features of type-II systems and are attributed to a combination of band bending and capacitive Coulomb charging although the latter is more important in GaSb/GaAs QDs and QRs ${ }^{13}$. We note that the peak emission energies observed in figure 2 predominately correspond to emission from the QR heavy-hole ground state. Recombination from excited heavy-hole states and light-hole states are likely to be present in the PL, but not resolved. 
(a)

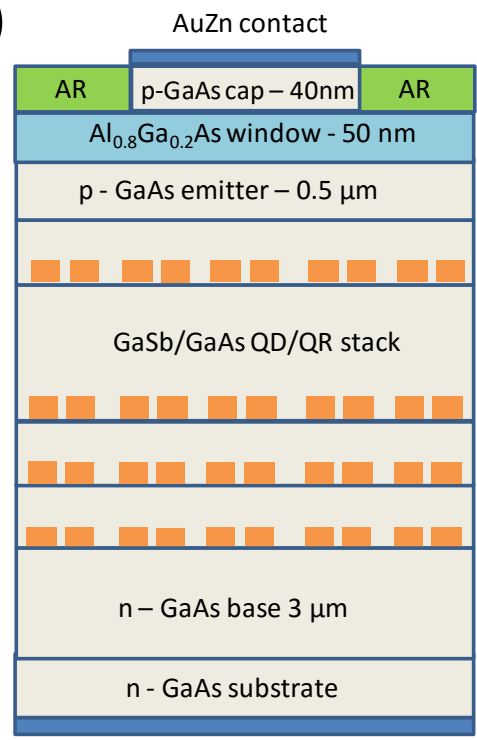

(c)

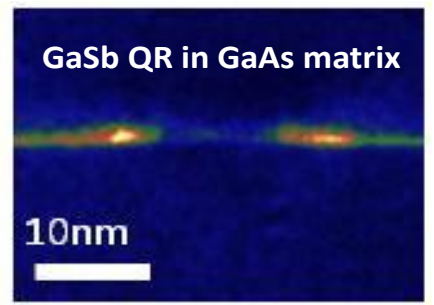

(b)

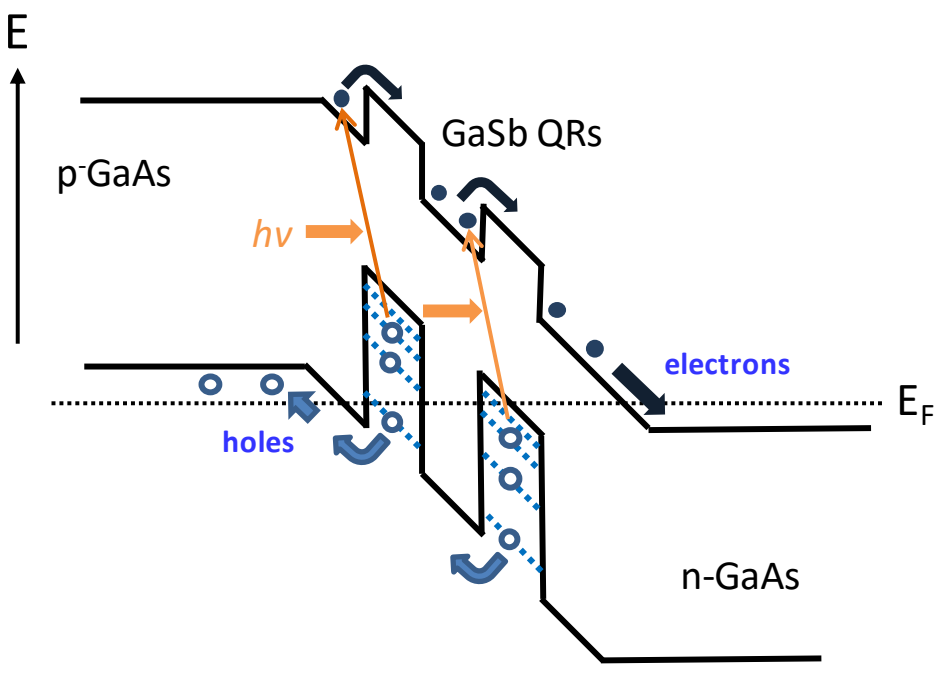

(e)

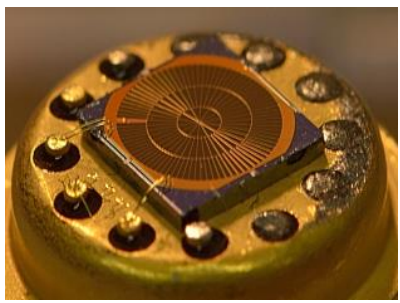

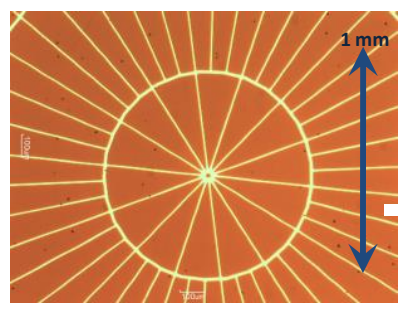

Figure 1 (a) Schematic of the GaSb/GaAs QR solar cell structure grown by MBE. (b) Schematic energy band-diagram of the structure. Absorption of photons with energy below the band gap of GaAs excites electrons from the discrete states in the GaSb QRs into the GaAs matrix. The photogenerated electron hole pairs are then separated under the influence of the built in electric field. (c) Transmission electron microscopy (TEM) of a single QR. (d) Image of the fabricated (3.5 mm diameter) solar cell mounted on a T0-5 header. (e) Image taken using an optical microscope showing the circular metal grid design.

(a)

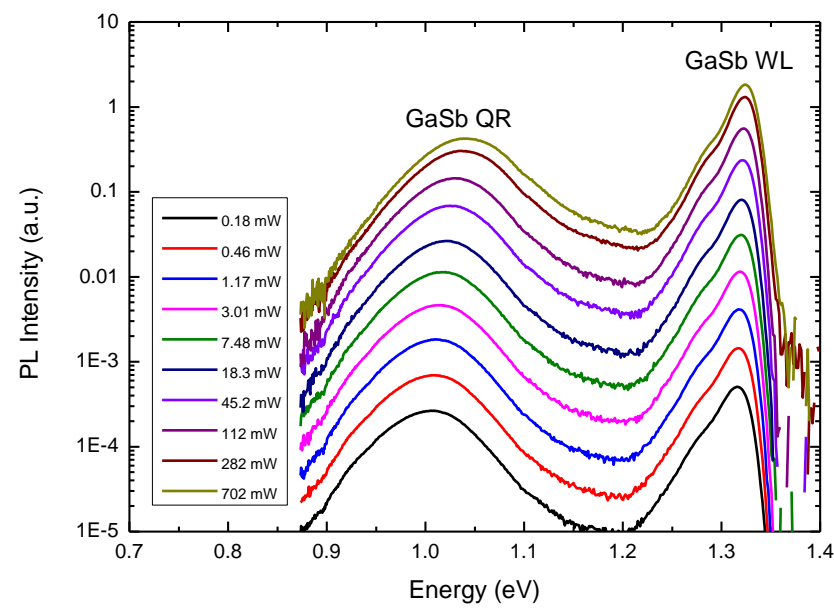

(b)

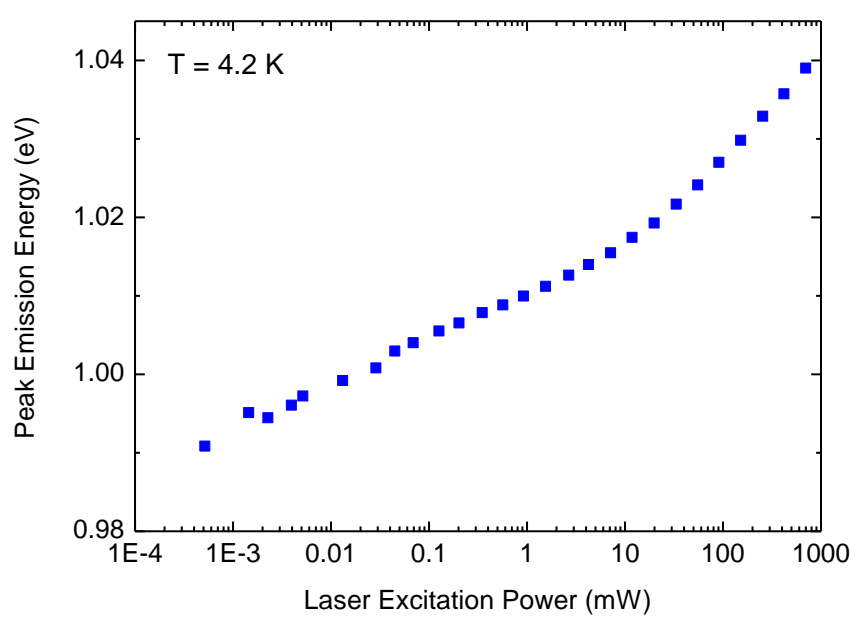

Figure 2: (a) Power Dependent Photoluminescence spectra measured at $4.2 \mathrm{~K}$ where the transitions corresponding to the GaSb/GaAs quantum rings and wetting layer are identified. (b) QR PL peak energy as a function of laser power. 
Figure 3 shows the temperature dependent PL spectra using high laser power $(700 \mathrm{~mW})$. The WL peak quickly quenches with temperature whilst the GaSb QRs exhibit strong PL emission up to room temperature, confirming their integrity and structural perfection. Figure $3 \mathrm{a}$ is an Arrhenius plot of the same data from which an activation energy of $240 \mathrm{meV}$ was obtained. This energy is attributed to the leakage of holes out of the QR via the WL and is considered to be the main process responsible for the quenching of the luminescence.

(a)

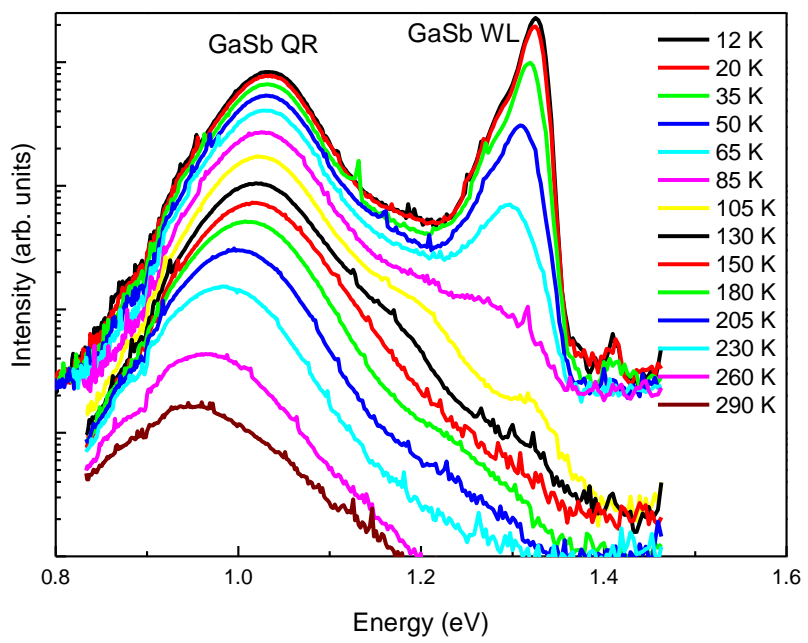

(b)

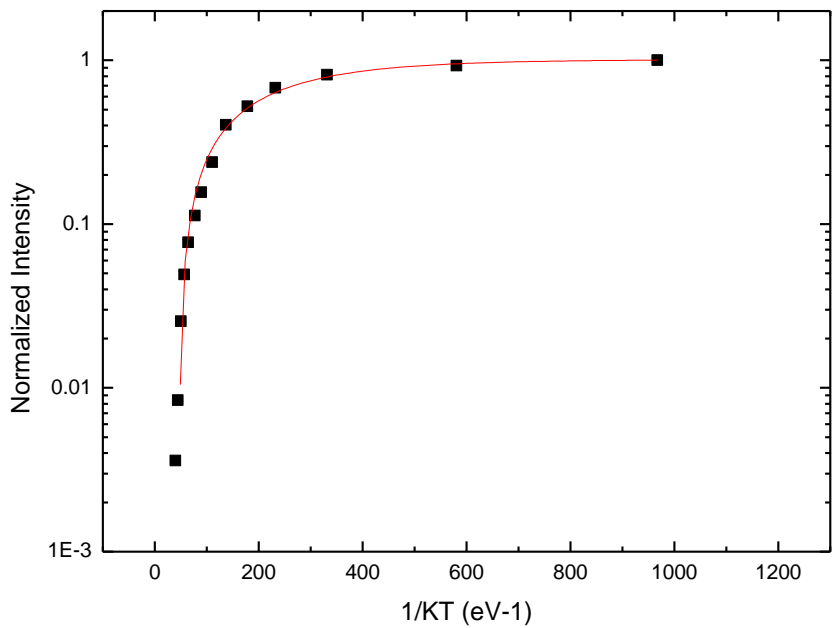

Figure 3: (a) Temperature Dependent Photoluminescence spectra (b) Arrhenius plot from which an activation energy of $240 \mathrm{meV}$ is obtained.

\section{GaSb/GaAs Quantum Ring Solar Cells}

Figure 4a shows the room temperature dark current density $(\mathrm{J})$ versus voltage $(\mathrm{V})$ characteristics measured from the QR SCs (solid lines). The linear parts of the dark J-V curves are fitted (dashed lines) using the standard diode equation

$$
J=J_{0}\left(\exp \left(\frac{q\left(V-I R_{S}\right.}{n k T}\right)-1\right)
$$

where $\mathrm{J}_{0}$ is the reverse saturation current, $\mathrm{q}$ the elementary charge, $\mathrm{R}_{\mathrm{s}}$ the series resistance, $n$ the ideality factor, $\mathrm{k}$ the Boltzmann constant and $\mathrm{T}$ the temperature. The fitting parameters are listed in Table I. The reverse saturation current for the GaAs control is $7.3 \times 10^{-12} \mathrm{~A} / \mathrm{cm}^{2}$ and the ideality factor is 1.7 , indicating that generation/recombination current dominates. An increase in the dark and reverse saturation currents is observed in the QR solar cells due to the introduction of additional recombination paths via the QR states. For the GaAs control cell unusual humps are observed at low voltage $(0-0.5 \mathrm{~V})$ which cannot be fitted using the standard diode equation. This behavior has been observed previously in GaAs ${ }^{14}$ and silicon SCs and was attributed to a high edge/surface recombination current. This is in contrast to the dark J-V curves from the QR SCs where no such humps are observed. This indicates that similar to InGaAs/InAs ${ }^{15}$ QDs the use of $\mathrm{GaSb} / \mathrm{GaAs} \mathrm{QRs}$ enables one to suppress lateral current flow to the device perimeter thereby reducing edge recombination, making QR highly suitable for small area concentrator solar cells. Figure 4b shows the illuminated 1 sun IV characteristics for the QR and GaAs control solar cells. Table II lists the sample details, open circuit voltage $\left(\mathrm{V}_{\mathrm{oc}}\right)$, short-circuit current $\left(\mathrm{J}_{\mathrm{sc}}\right)$, fill factor $(\mathrm{FF})$ and efficiency for each SC. The short-circuit current density $\left(J_{s c}\right)$ is enhanced by $5.9 \%$ in the QR cell due to the additional absorption from sub-bandgap photon absorption. However, a reduced open-circuit voltage $\left(V_{o c}\right)$ was observed, which is caused by the increased recombination current via the QRs as indicated by the dark current characteristics. 
(a)

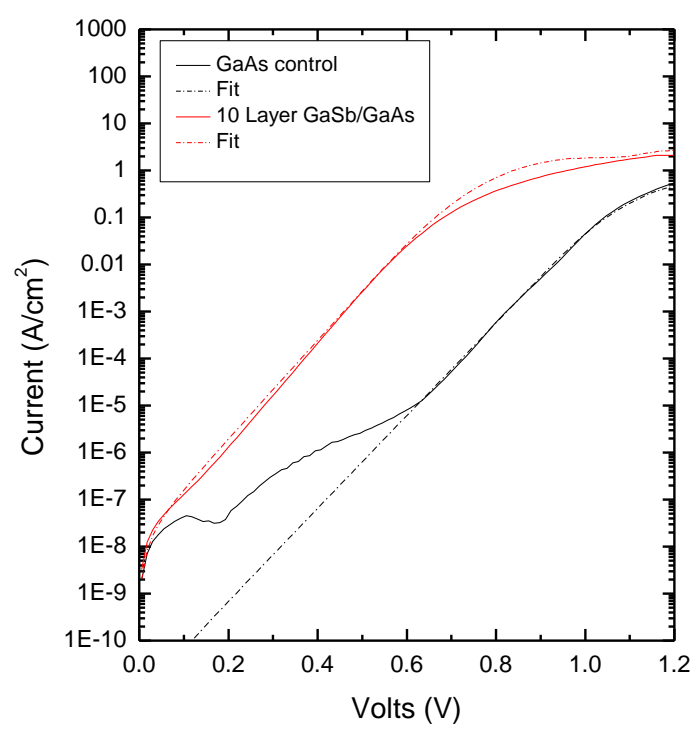

(b)

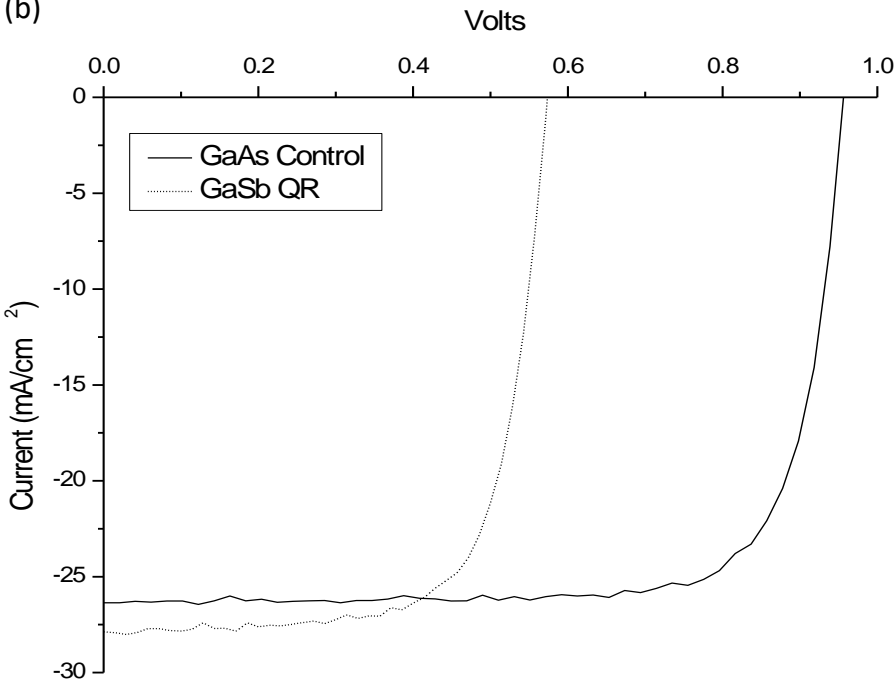

Figure 4a. Room temperature dark current density (J) versus forward bias (V) characteristics measured from the solar cells (solid lines) and fitted (dashed lines) using the standard diode equation. The non-ideal behavior for the GaAs control cell at low bias $(<0.6 \mathrm{~V})$ is characteristic of surface recombination which is suppressed in the QR solar cells. The tail-off at high voltages is due to series resistance. Figure 4b. Current density-voltage curves for the GaAs control and GaSb/GaAs QR solar cells obtained using 1 sun AM 1.5 illumination. The short-circuit current is enhanced by $5.9 \%$ in the solar cell containing 10 layers of QRs.

I

\begin{tabular}{|l|l|l|l|}
\hline Sample & $J_{0}\left(\mathrm{~A} / \mathrm{cm}^{2}\right)$ & $n$ & $R_{\mathrm{S}}(\Omega)$ \\
\hline GaAs control & $7.29 \times 10^{-12}$ & 1.7 & 1.7 \\
\hline 10 Layer $\mathrm{SC}$ & $1.56 \times 10^{-8}$ & 1.6 & 2.0 \\
\hline
\end{tabular}

II

\begin{tabular}{|c|c|c|c|c|}
\hline Sample & $\begin{array}{c}\mathrm{FF} \\
(\%)\end{array}$ & $\mathrm{J}_{\mathrm{sc}}\left(\mathrm{mA} / \mathrm{cm}^{2}\right)$ & $\begin{array}{c}\mathrm{V}_{\mathrm{oc}} \\
(\mathrm{V})\end{array}$ & $\begin{array}{c}\text { Efficiency } \\
(\%)\end{array}$ \\
\hline $\begin{array}{c}\text { GaAs } \\
\text { control }\end{array}$ & 78 & 26.4 & 0.96 & 19.7 \\
\hline $\begin{array}{c}10 \text { Layer } \\
\text { SC }\end{array}$ & 70 & 27.9 & 0.58 & 11.4 \\
\hline
\end{tabular}

Table I: Dark I-V characteristics. The table indicates the fitting parameters: $\mathrm{J}_{0}$ (reverse saturation current density), $\mathrm{n}$ (the ideality factor) and $R_{s}$ the series resistance. Table II: Measured 1 sun solar cell characteristics including FF (fill factor), $\mathrm{J}_{\mathrm{sc}}$ (the short-circuit current density), $\mathrm{V}_{\text {oc }}$ (open-circuit voltage) and efficiency.

Figure 5 shows the temperature dependence of the short-circuit current density $\left(J_{S C}\right)$ and open-circuit voltage $\left(V_{O C}\right)$ under 19 suns. Both the QR sample and the control sample show a constant increase of $J_{S C}$ with increasing temperature, resulting from the increased photo-absorption associated with the GaAs bandgap narrowing. At low temperature $(100 \mathrm{~K}), J_{S C}$ for the GaSb QR cell is lower than that of the GaAs control. However, $J_{S C}$ starts to increase rapidly above $180 \mathrm{~K}$, and surpasses the GaAs control cell above $250 \mathrm{~K}$. This behaviour is consistent with the observation that the photo-current due to subbandgap photon absorption is dominated by thermionic emission of holes. At the same time, $V_{o c}$ for the GaSb QR cell shows a slight change of slope, resulting in a net reduction of the efficiency. Figure 6 shows the external quantum efficiency (EQE) for the SCs (without anti-reflection (AR) coatings) at 100 and $290 \mathrm{~K}$. Although the 

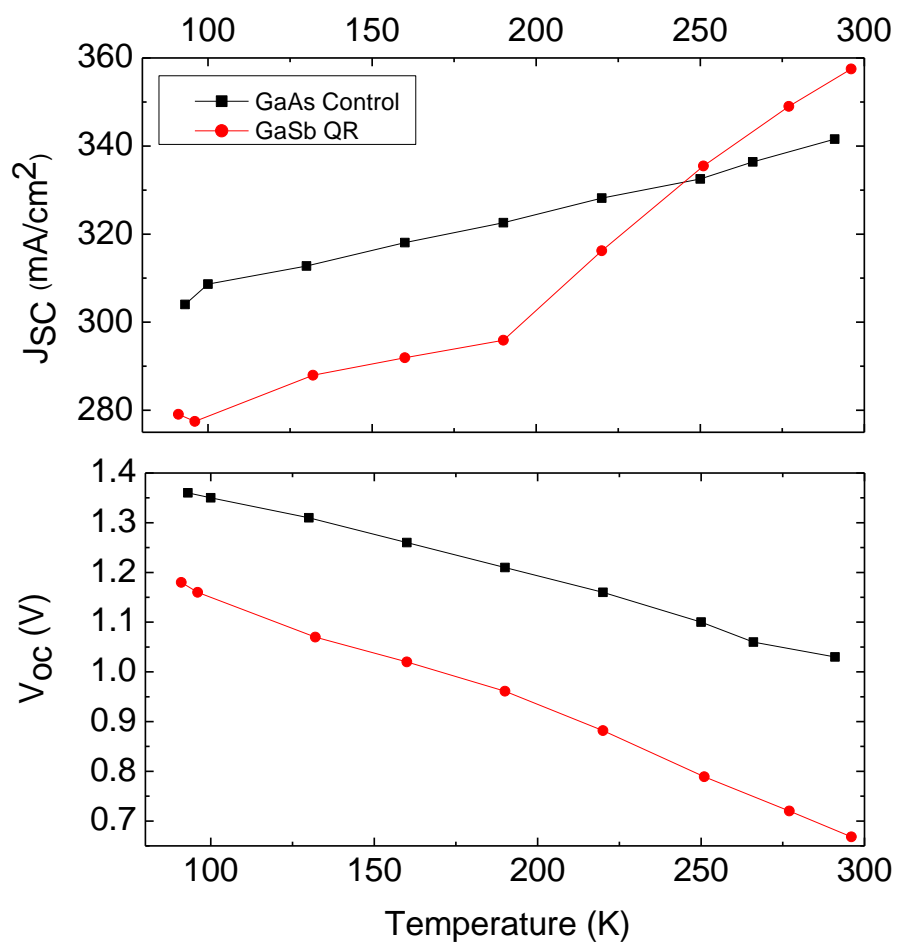

Figure 5: Temperature dependent device characteristics measured from the devices: short-circuit current density $\left(\mathrm{J}_{\mathrm{sc}}\right)$, open-circuit voltage $\left(\mathrm{V}_{\mathrm{oc}}\right)$.

(a)

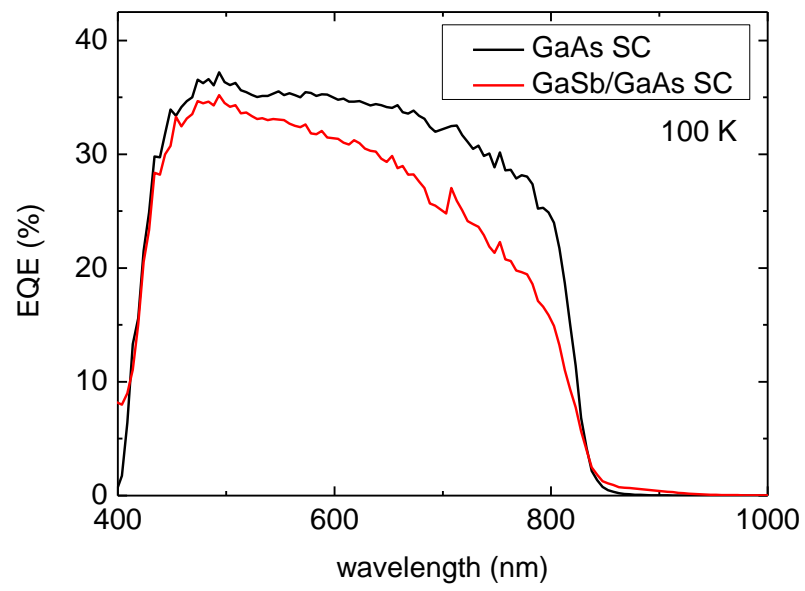

(b)

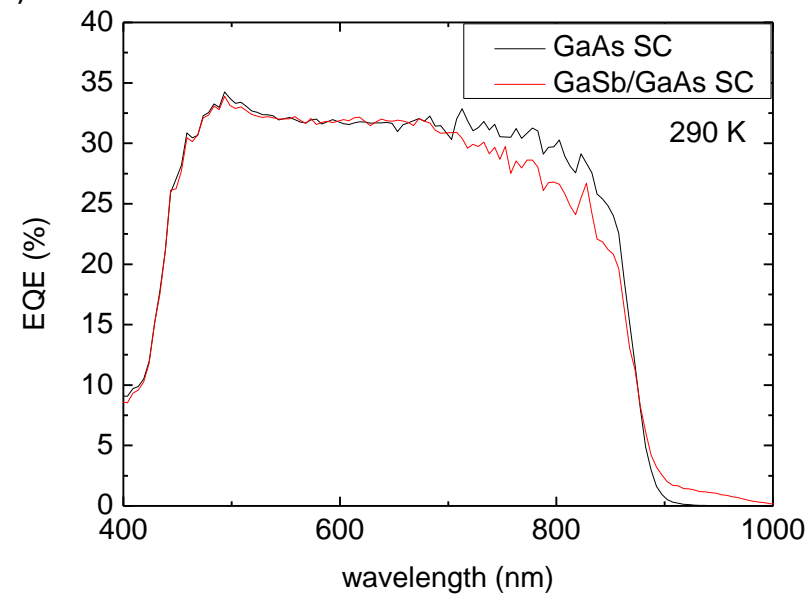

Figure 6 EQE for the GaAs control and GaSb QR solar cells at temperatures of (a) $100 \mathrm{~K}$ and (b) $290 \mathrm{~K}$.

QR solar cells show enhanced absorption below the GaAs bandgap, the EQE is substantially reduced above the band-gap at low temperatures. Here photo excited minority carriers (holes) which are generated in the $n$-base region diffuse towards the $p$-GaAs top via the intrinsic region. Due to the large valence band offset at the GaAs/GaSb QR interface, some of these holes become trapped by the QRs and recombine decreasing the open-circuit voltage and photocurrent. At room temperature, there is a smaller reduction in the above band-gap EQE consistent with increased thermal hole emission as also evident from the $\mathbf{J}_{\mathrm{sc}}$ increase. However, this decrease suggests that the thermionic hole emission might still be 
insufficient at room temperature. Therefore in order to further improve the solar cell performance, hole capture by QRs must be reduced, and/or the hole extraction rate from the QRs must be increased.

\section{Conclusion}

In summary, we have reported the successful MBE growth and fabrication of ten stacked layers of type II GaSb/GaAs QR SCs which show improved efficiency at longer wavelengths extending into the near-infrared up to $1400 \mathrm{~nm}$. A rapid increase of $\mathbf{J}_{\mathrm{sc}}$ was observed above $180 \mathrm{~K}$ due to the increase of the thermionic emission of holes. At room temperature a $5.9 \%$ increase in short-circuit current over the GaAs control cell was observed at 1 sun. A decrease in the open-circuit voltage to around $0.6 \mathrm{~V}$ is observed due to additional recombination paths introduced via the QRs.

\section{Acknowledgements}

We are grateful to Dr. R. Beanland and Dr. Ana Sanchez, University of Warwick, UK (TEM), Dr R. Airey and Dr. K. Kennedy, III-V National Centre, Sheffield, UK (processing). Financial support for this work was provided from EPSRC (grant EP/G070334/1) and The EU Marie-Curie Training networks PROPHET (FP7 - 264687) and PROMIS (H2020MSCA-ITN-2014-641899). P. J. Carrington and A. R. J. Marshall gratefully acknowledge support as fellowships from the Royal Academy of Engineering.

\section{References}

1. Luque A, Marti A. Increasing the efficiency of ideal solar cells by photon induced transitions at intermediate levels. Phys Rev Lett. 78(26):5014-7 (1997).

2. Richards BC, Lin Y, Patel P, Chumney D, Sharps PR, Kerestes C, et al. Performance and Radiation Resistance of Quantum Dot Multi-Junction Solar Cells. Ieee Phot Spec Conf.158-61. (2013).

3. Hubbard SM, Cress CD, Bailey CG, Raffaelle RP, Bailey SG, Wilt DM. Effect of strain compensation on quantum dot enhanced GaAs solar cells. Appl Phys Lett.;92(12) (2008)

4. Linares PG, Lopez E, Ramiro I, Datas A, Antolin E, Shoji Y, et al. Voltage limitation analysis in strain-balanced InAs/GaAsN quantum dot solar cells applied to the intermediate band concept. Sol Energ Mat Sol C. 132:178-82. (2015) 5. Sugaya T, Furue S, Komaki H, Amano T, Mori M, Komori K, et al. Highly stacked and well-aligned In0.4Ga0.6As quantum dot solar cells with $\mathrm{In}_{0.2} \mathrm{Ga}_{0.8}$ As cap layer. Appl Phys Lett. 97(18) (2010)

6. Ramiro I, Antolin E, Steer MJ, Linares PG, Hernandez E, Artacho I, et al. InAs/AlGaAs quantum dot intermediate band solar cells with enlarged sub-bandgaps. 2012 38th Ieee Photovoltaic Specialists Conference (Pvsc). 2012.

7. Kim YH, Ban KY, Zhang CM, Honsberg CB. Material and device characteristics of InAs/GaAsSb sub-monolayer quantum dot solar cells. Appl Phys Lett. 107(15) (2015)

8. Wagener MC, Carrington PJ, Botha JR, Krier A. Evaluation of the two-photon absorption characteristics of $\mathrm{GaSb} / \mathrm{GaAs}$ quantum rings. Journal of Applied Physics.116(4) (2014)

9. Wagener MC, Carrington PJ, Botha JR, Krier A. Simulation of the enhanced infrared photoresponse of type-II $\mathrm{GaSb} / \mathrm{GaAs}$ quantum ring solar cells. Appl Phys Lett. 103(6). (2013)

10. Carrington PJ, Wagener MC, Botha JR, Sanchez AM, Krier A. Enhanced infrared photo-response from GaSb/GaAs quantum ring solar cells. Appl Phys Lett. 101(23) (2012)

11. Hwang J, Martin AJ, Millunchick JM, Phillips JD. Thermal emission in type-II GaSb/GaAs quantum dots and prospects for intermediate band solar energy conversion. Journal of Applied Physics.111(7). (2012)

12. Carrington PJ, Young RJ, Hodgson PD, Sanchez AM, Hayne M, Krier A. Long-Wavelength Photoluminescence from Stacked Layers of High-Quality Type-II GaSb/GaAs Quantum Rings. Cryst Growth Des.;13(3):1226-30. (2013)

13. Hodgson PD, Young RJ, Kamarudin MA, Zhuang QD, Hayne M. Hole migration and optically induced charge depletion in GaSb/GaAs wetting layers and quantum rings. Phys Rev B.;88(15). (2013)

14. Gu TY, El-Emawy MA, Yang K, Stintz A, Lester LF. Resistance to edge recombination in GaAs-based dots-ina-well solar cells. Appl Phys Lett.;95(26). (2009)

15. Lu HF, Fu L, Jolley G, Tan HH, Tatavarti SR, Jagadish C. Temperature dependence of dark current properties of InGaAs/GaAs quantum dot solar cells. Appl Phys Lett.98(18). (2011) 